\title{
Manifestation of quark-hadron duality via the Adler D-function
}

\section{O. Solovtsova*}

Joint Institute for Nuclear Research, 141980 Dubna, Russia

Gomel State Technical University, 246746 Gomel, Belarus

E-mail: olsoletheor.jinr.ru

\begin{abstract}
Analyzing the hadronic contributions to various physical quantities within the method based on the variational perturbation theory we obtain that the suggested method allows us to describe well the Adler function corresponding to the $e^{+} e^{-}$annihilation and the $\tau$-decay into hadrons, the hadronic contributions to the lepton anomalous magnetic moment, and to the running electromagnetic fine structure constant. A common feature of all these quantities is that their theoretical description contains the vector current correlator and includes the low-energy region with the scale about $1 \mathrm{GeV}$ and less in which the standard perturbation theory cannot be applied. We discuss the reason for such good agreement and formulate a criterion that is satisfied by the considered quantities. It is shown that such consent may be understood if we use the concept of quark-hadron duality. If the method supports the required analytic properties, the criterion obtained ensures the equivalence of the transition from the timelike to the spaselike region.
\end{abstract}

The XXI International Workshop High Energy Physics and Quantum Field Theory,

June 23 - June 30, 2013

Saint Petersburg Area, Russia

${ }^{*}$ Speaker. 


\section{Intoduction}

To compare theoretical results with experimental data, one often uses the concept of quark-hadron duality, which establishes a bridge between quarks and gluons, a language of theoreticians, and real measurements with hadrons performed by experimentalists. The idea of quark-hadron duality was formulated in the paper by Poggio, Quinn, and Weinberg [1] as follows: Inclusive hadronic cross sections, once they are appropriately averaged over an energy interval, must approximately coincide with the corresponding quantities derived from the quark-gluon picture. To check direct consequences of the theory without using model assumptions, it is important to connect measured quantities with the "simplest" theoretical objects. Some single-argument functions, which include the Adler $D$-function [2], directly related to the experimentally measured quantities can play the role of these objects.

In this report, we concentrate on the $D$-function, corresponding to the $e^{+} e^{-}$annihilation and the $\tau$-decay into hadrons, the hadronic contribution to the anomalous magnetic moment of the leptons, and the hadronic contribution to the electromagnetic coupling. As can be seen from the following formulas, these physical quantities and functions are expressed through the experimentally measured function $R(s)=\operatorname{Im} \Pi(s) / \pi$, the imaginary part of the vector current correlator $\Pi(s)$, integrated with some known function:

- the Adler function

$$
D\left(Q^{2}\right)=-Q^{2} \frac{d \Pi\left(-Q^{2}\right)}{d Q^{2}}=Q^{2} \int_{0}^{\infty} d s \frac{R(s)}{\left(s+Q^{2}\right)^{2}}
$$

- the hadronic contribution to the anomalous magnetic moment of the lepton (in the leading order in electromagnetic coupling constant)

$$
a_{l}^{\mathrm{had}}=\frac{1}{3}\left(\frac{\alpha}{\pi}\right)^{2} \int_{0}^{\infty} \frac{d s}{s} K_{l}(s) R(s),
$$

where $l=\mu, e, \tau$, and $K_{l}(s)$ is a known QED kernel function;

- the strong interaction contribution to the running of the fine structure constant at $M_{Z}$

$$
\Delta \alpha_{\mathrm{had}}^{(5)}\left(M_{Z}^{2}\right)=-\frac{\alpha(0)}{3 \pi} M_{Z}^{2} \mathscr{P} \int_{0}^{\infty} \frac{d s}{s} \frac{R(s)}{s-M_{Z}^{2}} .
$$

By definition, all these quantities and functions include an infrared region as part of the interval of integration and, therefore, they cannot be directly calculated within perturbative QCD.

The approach that we use here to describe the quantities and functions mentioned above is based on the nonperturbative expansion method $[3,4]$. In the case of QCD the method leads to a new small expansion parameter. Even going into the infrared region of small momenta, where the running coupling becomes large and the standard perturbative expansion fails, the nonperturbative expansion parameter remains small and the approach holds valid. 


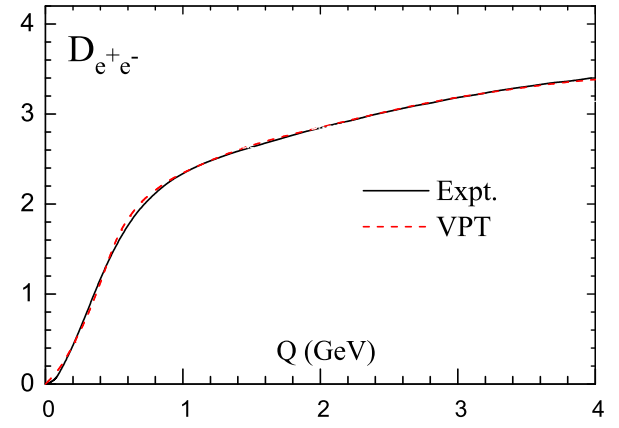

Figure 1: The Adler function for the $e^{+} e$ annihilation into hadrons. The solid curve is the VPT result for five active quarks. The experimental curve is taken from Ref. [12].

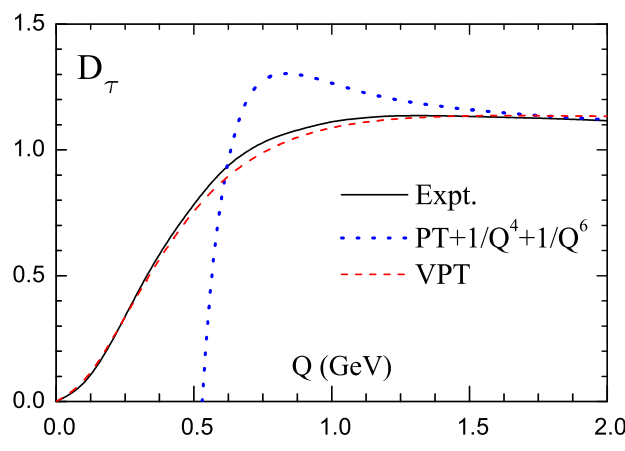

Figure 2: The 'light' Adler function. The experimental curve (solid line) and the perturbative result with power corrections (dotted line) are taken from Ref. [6].

\section{The Adler function}

Let us remind that the cross-section for the process of $e^{+} e^{-}$annihilation into hadrons, or the Drell ratio $R(s)$, the normalized hadronic cross-section, is a physically measured quantity defined for the timelike momentum transfer, and at the low energy scale has a resonance structure which is difficult to describe without model considerations. At the same time, the Adler $D$-function is determined by expression (1.1) in the Euclidean region (for the spacelike momentum transfer), $Q^{2}=-q^{2}>0$, is a smooth function without traces of the resonance structure. S. Adler observed this many years ago [2] and, consequently, he suggested using the spacelike function which was the first derivative of the function $R(s)$. The Adler $D$-function is a really useful object (see Ref. [5] for more details) and was studied in many papers $[6,7,8,9,10,11,12,13,14,15,16,17,18]$.

Figures 1 and 2 show the $Q^{2}$-behavior of the Adler function. Figure 1 shows the $D$-function corresponding to the process of $e^{+} e^{-}$annihilation into hadrons. ${ }^{1}$ The rich and precision experimental data on the $\tau$ lepton decays into hadrons provide a unique opportunity to test QCD at low energy scale and, in particular, for construction of the $D_{\tau}$ function. Figure 2 shows the 'light' Adler function corresponding to the non strange vector channel of $\tau$ decay data [19, 20]. Figure 2 also shows the perturbative result with power corrections (dotted line), which was taken from Ref. [6]. These figures demonstrate that indeed the Adler function turned out to be a smooth function without traces of the resonance structure of the timelike function $R(s)$. One can see that theoretical curves that have been found within the method based on the variational perturbation theory (VPT) are in good agreement with the experimental ones down to the lowest energy scale.

Note that any finite order of the operator product expansion fails to describe the infrared tail of the $D$ function (see dotted line in Fig. 2). Within the VPT approach, the nonperturbative expansion parameter $a$ remains small even going into the infrared region of small momenta where the running coupling becomes large and the approach holds valid.

\footnotetext{
${ }^{1}$ We grateful to F. Jegerlehner who supply us with the corresponding data.
} 


\section{Method and numerical results}

Let us briefly recall the essential features of the method that we use here to describe the functions connected with $R(s)$ mentioned in the Introduction. The method we use to construct a description of the $R$-related quantities is the VPT [3,4]. Within this approach, a quantity under consideration is represented in the form of the so-called floating or variational series. A certain variational procedure is combined with the possibility of calculating corrections to the principal contribution, which allows the possibility of probing the validity of the leading contribution and the region of applicability of the results obtained. The VPT series is different from the conventional perturbative expansion and can be used to go beyond the weak-coupling regime. This allows one to deal with considerably lower energies than in the case of perturbation theory. It is important to note, that the VPT approach maintains the required analytical properties of the Adler function. As can be seen from Eq. (1.1), the function $D\left(Q^{2}\right)$ is the analytic function in the complex $Q^{2}$-plane with the cut along the negative real axis. ${ }^{2}$ Next, by using the VPT approach it was formulated the model [23]. This model also incorporates the summation of threshold singularities [24] and takes into account the nonperturbative character of the light quark masses (see Refs. [22, 25] for more details). Note that as the normalization point we use a value of the $R_{\tau}^{V}$-ratio of the hadronic to leptonic $\tau$ - decay widths obtained by the ALEPH collaboration [19].

For the hadronic contribution to the lepton anomalous magnetic moment in the leading order in the electromagnetic coupling constant we obtain the values:

$$
a_{\mu}^{\text {had }}=(702 \pm 16) \times 10^{-10}, a_{e}^{\text {had }}=(1.64 \pm 0.07) \times 10^{-12}, a_{\tau}^{\text {had }}=3.28 \times 10^{-6} .
$$

For comparison, we list the some recent values: $a_{\mu}^{\text {had }}=(694.9 \pm 4.3) \times 10^{-10}[26], a_{e}^{\text {had }}=(1.678 \pm$ $0.014) \times 10^{-12}[27], a_{\tau}^{\text {had }}=(3.375 \pm 0.037) \times 10^{-6}[28]$.

Our calculation for the hadronic correction to the electromagnetic fine structure constant at the $Z$-boson scale gives $\Delta \alpha_{\text {had }}^{(5)}\left(M_{Z}^{2}\right)=(279.9 \pm 4.0) \times 10^{-4}$ which is close to value extracted from data: $\Delta \alpha_{\text {had }}^{(5)}\left(M_{Z}^{2}\right)=(275.7 \pm 0.8) \times 10^{-4}[29]$.

\section{4. $R-D$ self-duality}

Let us turn to the hadronic correction to the muon. The expression (1.2) can be rewritten in terms of the $D$-function as follows [30]

$$
a_{\mu}^{\mathrm{had}}=\frac{1}{6}\left(\frac{\alpha}{\pi}\right)^{2} \int_{0}^{1} \frac{d x}{x}(1-x)(2-x) D\left(\frac{x^{2}}{1-x} m_{\mu}^{2}\right) .
$$

It should be emphasized that expressions (1.2) and (4.1) are equivalent due to the analytic properties of the correlator $\Pi\left(q^{2}\right)$. If one uses a method that does not maintain the required properties, these expressions will no longer be equivalent and will imply different results. This situation is similar to that which occurs in the analysis of the inclusive $\tau$-decay [31]. Within VPT this transformation is justified [32], and one can use equally well either expression (1.2) or expression (4.1).

\footnotetext{
${ }^{2}$ The method based on the analytic perturbation theory in QCD [21] also preserves the correct analytical properties and leads to very close results [22].
} 
Now we investigate the question: What can we say about the connection between kernels in (1.2) and (4.1) when these expressions are equivalent? Let us rewrite these expressions in a more general form:

$$
\begin{aligned}
Q_{M} & =\int_{0}^{\infty} \frac{d s}{s} M(s) R(s) \quad \text { for the Minkowskian (timelike) region, } \\
Q_{E} & =\int_{0}^{\infty} \frac{d t}{t} E(t) D(t) \quad \text { for the Euclidean (spacelike) region. }
\end{aligned}
$$

The $R-D$ self-duality means: $Q_{M}=Q_{E}$. The answer to the question is the connection between the kernels

$$
M(s)=s \int_{0}^{\infty} d t \frac{E(t)}{(s+t)^{2}} .
$$

According to this expression, $M(s)$ is an analytic function in the complex plane with a cut along the negative real axis. The inverse relation reads

$$
E(t)=-\frac{1}{2 \pi \mathrm{i}} \int_{t-\mathrm{i} \varepsilon}^{t+\mathrm{i} \varepsilon} \frac{d z}{z} M(-z) .
$$

Applying this result to the hadronic correction to the muon we get $R-D$ self-duality expressions:

$$
\begin{aligned}
& a_{\mu}^{\mathrm{had}}=\frac{1}{3}\left(\frac{\alpha}{\pi}\right)^{2} \int_{0}^{\infty} \frac{d s}{s} M(s) R(s), \\
& a_{\mu}^{\mathrm{had}}=\frac{1}{3}\left(\frac{\alpha}{\pi}\right)^{2} \int_{0}^{\infty} \frac{d t}{t} E(t) D(t),
\end{aligned}
$$

where $\quad M(s)=\eta^{2}\left(1-\frac{\eta^{2}}{2}\right)+(1+\eta)^{2}\left(1+\frac{1}{\eta^{2}}\right)\left[\ln (1+\eta)-\eta+\frac{\eta^{2}}{2}\right]+\frac{1+\eta}{1-\eta} \eta^{2} \ln \eta$

$$
E(t)=\frac{1}{2}\left[\frac{\sqrt{1+4 m_{\mu}^{2} / t}-1}{\sqrt{1+4 m_{\mu}^{2} / t}+1}\right]^{2}, \quad \eta=\frac{1-v}{1+v}, \quad v=\sqrt{1-\frac{4 m_{\mu}^{2}}{s}} .
$$

\section{Summary}

We have analyzed various physical quantities and functions generated by $R(s)$ based on the nonperturbative VPT-method (Adler functions, hadronic contributions to anomalous magnetic moments of leptons and so on). It was shown that the method allows us to describe these quantities well down to a low energy scale. We investigate the reason of such good agreement, and as a result we formulate the criterion which we called as the R-D self-duality. The quantities considered satisfy this criterion, and they can be described in terms of the Adler function, which is well described within this method.

This work was supported in part by the BelRFBR-JINR grant No. F12D-002 and the RFBR grant No. 11-01-00182. 


\section{References}

[1] E.C. Poggio, H.R. Quinn, and S. Weinberg, Phys. Rev. D 13 (1976) 1958.

[2] S.L. Adler, Phys. Rev. D 10 (1974) 3714.

[3] I.L. Solovtsov, Phys. Lett. B 327 (1994) 335; 340 (1994) 245.

[4] A.N. Sissakian and I.L. Solovtsov, Phys. Part. Nucl. 30 (1999) 461.

[5] S.L. Adler, Adventures in theoretical physics: Selected papers of Stephen L. Adler: Drafts of commentaries, hep-ph/0505177, $117 \mathrm{pp}$.

[6] S. Peris, M. Perrottet, and E. de Rafael, JHEP 05 (1998) 011.

[7] S. Eidelman, F. Jegerlehner, A.L. Kataev, and O. Veretin, Phys. Lett. B 454 (1999) 369.

[8] K.A. Milton, I.L. Solovtsov, and O.P. Solovtsova, Phys. Rev. D 64 (2001) 016005.

[9] A.N. Sisakian, I.L. Solovtsov, and O.P. Solovtsova, JETP Lett. 73 (2001) 166.

[10] A.E. Dorokhov, Phys. Rev. D 70 (2004) 094011.

[11] A.L. Kataev, Phys. Lett. B 668 (2008) 350.

[12] F. Jegerlehner, Nucl. Phys. Proc. Suppl. 181-182 (2008) 135.

[13] P.A. Baikov, K.G. Chetyrkin, and J.H. Kühn, Phys. Rev. Lett.101 (2008) 012002.

[14] A.V. Nesterenko, Nucl. Phys. Proc. Suppl. 186 (2009) 207.

[15] B.A. Magradze, Few Body Syst. 48 (2010) 143 [Erratum-ibid. 53 (2012) 365].

[16] A.L. Kataev and S.V. Mikhailov, PoS QFTHEP2010 (2010) 014.

[17] T. Goecke, C.S. Fischer, and R. Williams, Phys. Lett. B 704 (2011) 211.

[18] P.A. Baikov, K.G. Chetyrkin, J.H. Kühn, and J. Rittinger, Phys. Lett. B 714 (2012) 62.

[19] ALEPH Collaboration, R. Barate et al., Eur. Phys. J. C 4 (1998) 409.

[20] OPAL Collaboration, K. Ackerstaff et al., Eur. Phys. J. C 7 (1999) 571.

[21] D.V. Shirkov and I.L. Solovtsov, Phys. Rev. Lett. 79 (1997) 1209.

[22] K.A. Milton, I.L. Solovtsov, and O.P. Solovtsova, Mod. Phys. Lett. A 21 (2006) 1355.

[23] I.L. Solovtsov, Proceedings of the XVIII International Baldin Seminar on High Energy Physics Problems, Eds. A.N. Sissakian, V.V. Burov, A.I. Malakhov, JINR E1,2-2008-113, vol. I, p. 28.

[24] K.A. Milton and I.L. Solovtsov, Mod. Phys. Lett. A 16 (2001) 2213.

[25] O.P. Solovtsova, Nucl. Phys. Proc. Suppl. 189 (2009) 72.

[26] T. Teubner, K. Hagiwara, R. Liao, A. D. Martin, and D. Nomura, Nucl. Phys. Proc. Suppl. 225-227 (2012) 282.

[27] D. Nomura, T. Teubner, Nucl. Phys. B 876 (2013) 236.

[28] S. Eidelman, M. Giacomini, F.V. Ignatov, and M. Passera, Nucl. Phys. Proc. Suppl. 169 (2007) 226.

[29] S. Bodenstein, C. A. Dominguez, K. Schilcher, and H. Spiesberger, Phys. Rev. D 86 (2012) 093013.

[30] E. de Rafael, Phys. Lett. B 322 (1994) 239.

[31] K.A. Milton, I.L. Solovtsov, and O.P. Solovtsova, Phys. Lett. B 415 (1997) 104.

[32] H.F. Jones and I.S. Solovtsov, Phys. Lett. B 349 (1995) 519. 